\title{
Impact of Nanoparticles on Pathogenic Fungi
}

\author{
Josef Jampilek $^{1,2}$, Martin Pisarcik ${ }^{3}$ \\ ${ }^{1}$ Faculty of Natural Sciences, Comenius University, Ilkovicova 6, 84215 Bratislava, Slovakia; josef.jampilek@gmail.com \\ ${ }^{2}$ Regional Centre of Advanced Technologies and Materials, Faculty of Science, Palacky University, Slechtitelu 27, \\ 78371 Olomouc, Czech Republic \\ ${ }^{3}$ Faculty of Pharmacy, Comenius University, Kalinciakova 8, Bratislava, 83232 Slovakia; pisarcik@fpharm.uniba.sk
}

\section{Extended Abstract}

Fungi are simple eukaryotic organisms that colonize various environments around the planet. Higher fungi used in the kitchen, brewery, viticulture and folk medicine. Secondary metabolites of higher fungi and microscopic fungi can be the main structures for the design of biologically active ingrediences/drugs, used in biotechnology for production or biotransformation of various substances, for nanoparticle synthesis. Unfortunately, some fungi attack humans, animals and plants; It is estimated that 270,000 species of fungi are associated with plants and 325 are known to infect humans. Fungi cause diseases ranging from superficial and cutaneous forms through subcutaneous complicated forms to systemic (invasive fungal infections), often fatal diseases. Diseases are caused by colonization/proliferation/sporulation of fungi in tissues/body fluids or excretion of toxins. About 1.2 milliard people worldwide suffer from fungal diseases, but a significant proportion of these infections are difficult to diagnose and treat. Every year, 1.5 to 2 million people die of fungal infections worldwide. Thus fungal diseases are a worldwide public health problem; pose a serious threat to people with weakened immune systems (cancer, AIDS, transplantation, diabetes). The development of resistance to several systemically used drugs and the development of cross-resistant or multi-resistant strains are serious. In addition, most drugs are approved for the treatment of nail, skin and mucous membrane mycoses due to a narrow therapeutic window and limited bioavailability [1-4].

One of the reasons why the process of research and development of new antifungals is so complex is the fact that the eukaryotic nature of a fungal cell is very similar to that of a human cell. Therefore, it is very important to look for antifungal agents whose mechanism of action focuses on the specific structure of the fungal cell. The procedure for identifying agents with a new mode of action is relatively long and risky, and therefore the preparation of nanoparticles/nanoformulations of existing antifungal agents may be a first choice approach [1,3-8].

The goal of this contribution is to summarize the knowledge about nanosystems based on organic polymeric and inorganic matrices or hybrid materials as active antifungal agents.

This study was supported by the Slovak Research and Development Agency (Grant No. APVV-17-0373).

\section{References}

[1] J. Jampilek, "Potential of agricultural fungicides for antifungal drug discovery," Expert Opin. Drug Dis., vol. 11, no. 1, pp. 1-9, 2016.

[2] J. Jampilek, "How can we bolster the antifungal drug discovery pipeline?" Future Med. Chem., vol. 8, no. 12, pp. 13931397, 2016.

[3] J. Jampilek and K. Kralova, "Nano-antimicrobials: Activity, benefits and weaknesses," in: Nanostructures for Antimicrobial Therapy, A. Ficai, A.M. Grumezescu, Eds. Amsterdam: Elsevier 2017, pp. 23-54.

[4] J. Jampilek and K. Kralova, "Impact of nanoparticles on toxigenic fungi," in: Nanomycotoxicology - Treating Mycotoxins in Nano Way, M. Rai, K.A. Abd-Elsalam, Eds. London: Academic Press \& Elsevier, 2020, pp. 309-348.

[5] J. Jampilek and K. Kralova, "Nanocomposites: Synergistic nanotools for management mycotoxigenic fungi," in: Nanomycotoxicology - Treating Mycotoxins in Nano Way, M. Rai, K.A. Abd-Elsalam, Eds. London: Academic Press \& Elsevier, 2020, pp. 349-383.

[6] M. Pisarcik, et al. "Silver nanoparticles stabilised by cationic gemini surfactants with variable spacer length," Molecules, vol. 22, no. 10, pp. 1794, 2017. 
[7] M. Pisarcik, et al. "Silver nanoparticles stabilised with cationic single-chain surfactants. Structure-physical propertiesbiological activity relationship study," J. Mol. Liq., vol. 272, pp. 60-72, 2018.

[8] M. Pisarcik, et al. "Phosphonium surfactant stabilised silver nanoparticles. Correlation of surfactant structure with physical properties and biological activity of silver nanoparticles," J. Mol. Liq. vol. 314, 113683, 2020. 